\title{
A TV monitored system for recording open-field activity in the rat
}

\author{
P. J. LIVESEY and K. LEPPARD \\ University of Western Australia, Nedlands 6009, Western Australia
}

\begin{abstract}
A system for monitoring and recording the movements of a rodent in an open field is described. A TV camera and a grid generator combine the animal's position in the field with an overlaid 16 by 16 grid pattern. These are displayed on the TV monitor. The location of the animal is recorded with each sweep of the video raster. The movement of the animal is then plotted by a Rockwell microprocessor that prints out the animal's location at set intervals.
\end{abstract}

Since the open-field test was first introduced by Hall in 1934, various attempts have been made to develop a system that would automate the recording of the rat's movement in the field and remove the need for the experimenter's presence. Recent designs have relied on detection matrices in the floor of the field that register the movement of the animal across the floor (Pfister, Mudge, \& Harcombe, 1978; Porter, Hardy, \& Furber, 1979).

These detection units are usually in the form of pressure transducers or touch plates, and earlier models were subject to mechanical failure due to rat urine or feces. In a recent apparatus, this problem was obviated by the use of electrical grating touch-activated switches beneath a thin Plexiglas sheet (Porter et al., 1979). While such apparatuses have the advantage of automated scoring, they are still subject to mechanical failure and to the problem of not knowing what particular movement is being registered unless direct observation of the subject is undertaken.

The apparatus to be described is based on a TV monitor system that permits automatic recording of the animal's locomotion without the presence of an observer. It was developed from an apparatus devised by Lubinski, Dickson, and Cairns (1977) for converting TV scanning signals into $X Y$ coordinates for object tracking. The system permits either on-line observation of the animal's behavior on the TV monitor or subsequent viewing from videotape. There is no floor transducer system that might be subject to mechanical failure.

The core of the system is a grid generator that produces a square grid pattern. The video signal from the camera, showing the rat in the field, is then combined with the grid pattern on the monitor, thus locating the rat in one of the units of the 16 by 16 grid. The location is read off using $X, Y$ counters, this location is stored in

This project was supported by Grant A76/15232 from the Australian Research Grants Committee. Details of the crystal oscillator, divider network, and pulse shaper will be provided on request. a Rockwell microprocessor, and a record is printed out. The location of the rat is checked periodically (e.g., every $1 \mathrm{sec}$ ), and if it has changed, the new location is registered and printed out. Thus a continuous record of the animal's location and, therefore, of its movment through the field is achieved in terms of the 16 by 16 grid. Through computer link-up, various data analyses can be achieved (e.g., number of cell entries per unit of time, rate of locomotion, path of locomotion, percentage of wall following, etc.). A printout of the path taken can be achieved, as well. If further analysis of the rat's behavior in the field is desired, this can be observed from playback of the videotape. Details of the system follow.

\section{APPARATUS}

The open field is $1 \mathrm{~m}$ square with walls $53 \mathrm{~cm}$ high and with floor and walls painted mat black. Over the center of the field is a frame a further $81 \mathrm{~cm}$ high that carries the TV camera. The camera has an Australian Standard Sweep pattern of 625 lines/50 frames per second and external sync facilities.

The core of the system for generation of the 16 by 16 grid pattern and for location of the rat in the field is a $1-\mathrm{MHz}$ crystal oscillator with a divider network and pulse shaper. This produces three outputs: (1) $50-\mathrm{Hz}$ 200- to 250-microsec pulses to provide vertical synchronization, (2) 15,625- $\mathrm{Hz} 5$ microsec pulses for horizontal synchronization, and (3) $500 \mathrm{kHz}$, with differentiated and clipped pulses, for generation of the vertical lines of the grid pattern.

\section{Grid Generation}

A 16 by 16 grid pattern is generated in the center of the video monitor. This corresponds to the floor area of the open field as viewed by the camera. Generation of the grid as follows.

Vertical lines. Each horizontal line from the camera has a duration of 64 microsec. The $500-\mathrm{kHz}$ pulse generator produces bright spots along this line at 2 -microsec intervals. These bright spots, being in synchronization with the video raster from the camera, form vertical lines on the video display. 
A counter and gate network produces a square wave for the grid mixer. The counter registers the number of 2-microsec pulses along each horizontal line and, at the 8 th pulse, switches positive for 16 pulses ( 32 microsec) centrally in the 64 microsec available. This provides an enhanced brightness contrast, or bright up, for the central 17 vertical lines in the display.

Horizontal lines. A counter activates a line bright up every 19 lines from the camera, giving a series of horizontal lines on the video. A count is made of the bright horizontal lines, and, for the 17 lines in the center of the display, a positive square wave is produced and fed into the grid mixer.

These waveforms are combined to produce a 16 by 16 square grid pattern ready to be fed into the video mixer. The video signal from the camera is combined with the grid pattern to produce a picture of the subject overlaid with the grid. Levels are adjusted for optimal viewing. Video outside the grid is suppressed by the bright-up waveforms so that viewing is not complicated by stray images. This is illustrated in Figure 1.

\section{Subject Description}

By using a black background in the viewing area, the subject, a white rat, gives a good contrast, and detection is with a simple comparator. In this case, because of the low peak-to-peak value of the video signal (approximately $900 \mathrm{mV}$ ), an LM311 was used.

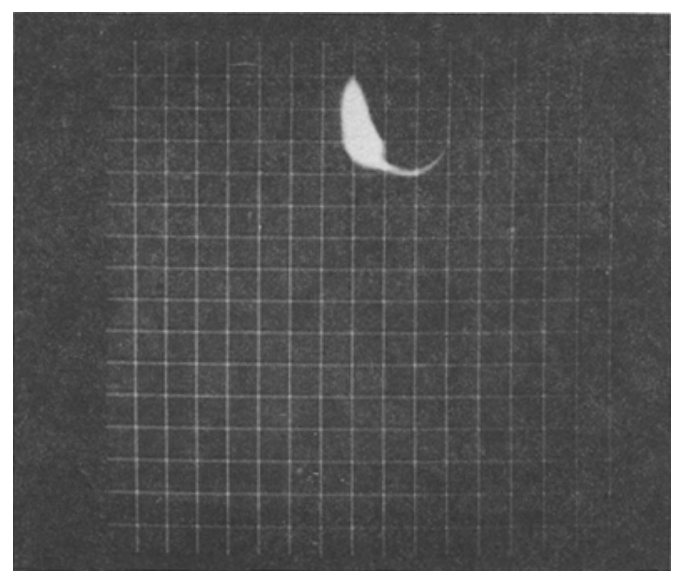

Figure 1. Video monitor showing 16 by 16 grid pattern and rat in the open field.

\section{Position Gate}

This network, under control by the grid pattern, allows the $X, Y$ counters to count the grid squares until the subject is detected on the screen. The $X, Y$ counters are stopped at the point of detection, and an interrupt signal is sent to the microprocessor to load the $\mathrm{X}, \mathrm{Y}$ coordinates directly from the counters. The position is thus recorded on every sweep of the video (50 per

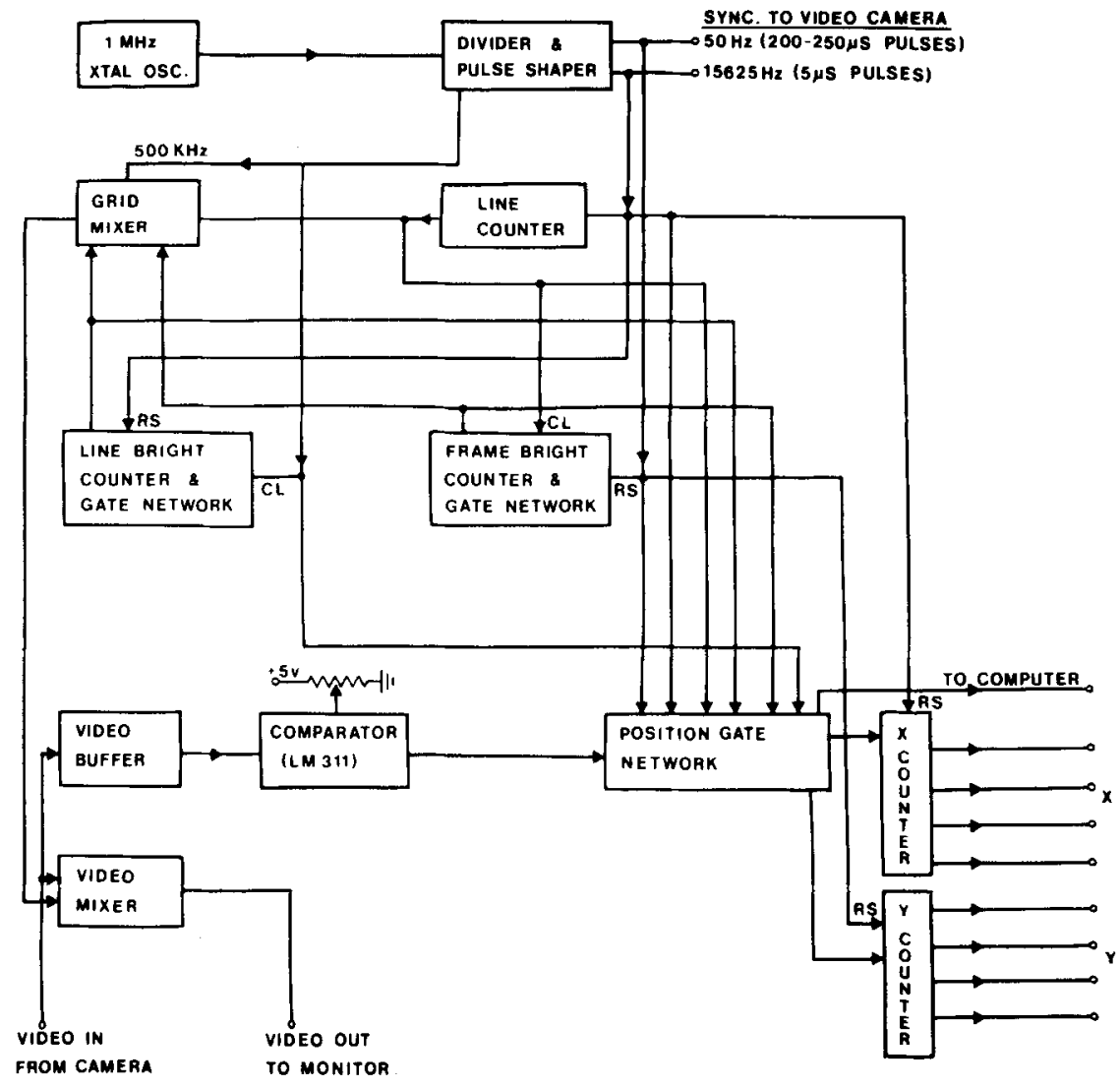

Figure 2. Block diagram of the recording system. 
second.). A block diagram of the system is shown in Figure 2.

The recorded movements of the animal within the field can be varied by software. With the position recorded on every sweep of the video, movement or oscillation of part of the rat's body (e.g., nose, tail, paw) across a line could generate spuriously high movement tallies. To overcome such a problem, the software program enables a comparison to be made after each sweep. If during a recording interval (e.g., $1.0 \mathrm{sec}$ ) the animal registers a movement into a new square and then back again, that movement is not recorded. Thus brief oscillations are ignored.

\section{REFERENCES}

Lubinski, K. S., Dickson, K. L., \& Cairns, J. Microprocessorbased interface converts video signals for object tracking. Computer Design, 1977, December, pp. 81-87.

Prister, H. P., Mudge, R. R., \& Harcombe, A. O. A multipurpose activity platform utilized in the open-field setting. Behavior Research Methods \& Instrumentation, 1978, 10, 21-22.

Porten, J. J., Hardy, J. J., \& Furber, A. M. A pressureactivated open-field apparatus for rodents. Behavior Research Methods \& Instrumentation, 1979, 11, 54-60.

(Received for publication June 15, 1981; accepted June 26, 1981.) 